International Journal of Pure and Applied Mathematics

Volume 100 No. 1 2015, 95-108

ISSN: 1311-8080 (printed version); ISSN: 1314-3395 (on-line version)

url: http://www.ijpam.eu

doi: http://dx.doi.org/10.12732/ijpam.v100i1.9

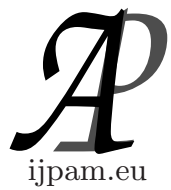

\title{
CONNECTIONS OF ZERO CURVATURE AND BÄCKLUND TRANSFORMATIONS
}

\author{
Paul Bracken \\ Department of Mathematics \\ University of Texas \\ Edinburg, TX78540, USA
}

\begin{abstract}
Zero curvature connections in a principal bundle are introduced. It is shown that such connections are very useful in formulating the Bäcklund problem and in constructing transformations. A general technique can be proposed for producing such transformations by defining the relevant connection coefficients appropriately. Finally, several applications of this work to various types of nonlinear partial differential equations will be presented.
\end{abstract}

AMS Subject Classification: 53Z05, 57R99, 55R10, 53B50

Key Words: connection, bundle, curvature, structure equations, Lax pair, Bäcklund

\section{Introduction}

The theory of differential systems has provided a very useful framework for investigating various types of partial differential equation. In particular, there exist connection forms which determine representations of zero curvature and these connections have been found to be especially effective in the study of nonlinear partial differential equations. It has been shown that such connections can produce Lax pairs as well as Bäcklund transformations in a very direct way. These types of connection have a special property which is crucial to this

Received: January 19, 2015

(c) 2015 Academic Publications, Ltd. url: www.acadpubl.eu 
program, namely, the curvature tensor of such a connection contains a subtensor which is directly proportional to the partial differential equation studied. For the case in which the connection tensor with these components vanishes, as on the lifts of solutions of a given nonlinear equation, it is said that the connection determines a representation of zero curvature.

Estabrook and Wahlquist as well as Hermann were some of the first to develop this field [1-4]. Hermann first introduced a particular connection which is of essentially of this basic type [5-6]. He proposed early on that the Bäcklund transformation could be interpreted as a connection similar in a certain sense to a connection which defines a representation of zero curvature. Hermann then formulated Bäcklund's problem as that of finding a section in a bundle space on whose pull back the Bäcklund connection is plane. These ideas have had a significant impact on the area of integrable systems [7-8].

The objective here is to go beyond this more primitive formulation by considering a jet-bundle $J^{r} E$ of $r$-jets over a lower dimensional bundle. Usually $r$ is taken to be two or three when second or third order equations are considered. The objective here is to review a more concise treatment of the formalism presented in [9] and to conclude with several examples to show how to apply it. It is hoped this will be useful more as a reference for applying these ideas to produce Lax pairs and Bäcklund transformations. The difficult step is to first write down an appropriate differential system which is relevant to a particular equation and also satisfies the right structure equations based on some Lie group. The relevant equations for using this information to construct Bäcklund transformations will be developed. A proposition is formulated to the effect that a connection in a principal bundle which defines a representation of zero curvature can lead to a system for the Bäcklund problem. A short review of the mechanics of doing this is given. Finally, a number of equations will be discussed in detail which will clearly indicate how results can be obtained from this method.

\section{Geometrical Formulation}

Connections which define representations of zero curvature provide a systematic way of investigating nonlinear partial differential equations [10-12]. Moreover, they provide a tool with which such objects as Lax pairs and Bäcklund transformations can be constructed for partial differential equations. The theory will be formulated in a general way for these kinds of equations which may have 
derivatives of arbitrary order and take the general form

$$
F\left(x^{i}, u, u_{j}, u_{j k}, u_{j k l}, \ldots\right)=0 .
$$

By working over a particular manifold which will be introduced shortly, equations of the form (1) can be represented in terms of a more general system of coordinates as

$$
F\left(x^{i}, u, \lambda_{j}, \lambda_{j k}, \lambda_{j k l}, \ldots\right)=0,
$$

The $\left\{x^{i}, u\right\}$ in $(2)$ are adapted local coordinates in the $(n+1)$-dimensional bundle $E$ over the $n$-dimensional base manifold $M$. The local coordinates of the base manifold $M$ are given as $\left\{x^{i}\right\}$ with $i, j, k=1, \cdots, n$ and for the examples here $n=\operatorname{dim} M=2$. The pair of coordinates can then be written as $\{x, y\}$. The larger manifold over which (2) is defined is the jet space $J^{r} E$, the space of holonomic $r$-jets of the local sections of the manifold $E$ [13]. It carries the system of coordinates $\left\{\lambda_{j_{1}, \cdots, j_{k}}\right\}$ where $k=1, \cdots, r$. Thus there exists the following sequence of inclusions $M \subset E \subset J^{r} E$.

It will then be important in due course to evaluate appropriate sections in these bundles. For any section $\Sigma \subset E$ which is defined by the equation $u=u\left(x^{1}, \cdots, x^{n}\right)$, the sections in $\Sigma^{r} \subset J^{r} E$ are defined by the equations

$$
u=u\left(x^{1}, \cdots, x^{n}\right), \quad \lambda_{j_{1}, \cdots, j_{k}}=u_{j_{1}, \cdots, j_{k}}, \quad k=1, \ldots, r,
$$

where the subscripts on $u$ on the right denote partial differentiation. Thus when this is done, (2) maps down onto (1). It is the intention here to show that there is a close relationship between connections which define representations of zero curvature and the equations of the Bäcklund or inverse problem.

There is the following generalization of the zero curvature theorem with respect to the bundle $P\left(J^{r} M, G\right)$. Here $G$ will be the group $G l(n)$ or a subgroup, however, other groups may be considered [14-15].

Theorem 2.1. Let a connection in the principal bundle $P\left(J^{r} M, G\right)$ be specified with $G$ a group which defines a representation of zero curvature corresponding to an equation of the type (1). A system of equations for the Bäcklund problem can be formulated for the equation.

Proof. Let

$$
\tilde{\omega}_{j}^{i}=\omega_{j}^{i}+\Gamma_{j \lambda}^{i} \omega^{\lambda}
$$

be connection forms in the principal bundle $P\left(J^{r} M, G\right)$ which defines a representation of zero curvature for (1). This connection which is defined in the principal bundle $P\left(J^{r} M, G\right)$ generates a connection in the associated prolonged 
bundle $\mathcal{F}\left(P\left(J^{r} M, G\right)\right)$. The connection forms in the bundle $\mathcal{F}\left(P\left(J^{r} M, G\right)\right)$ which correspond to the connection in $P\left(J^{r} M, G\right)$ can be specified by means of

$$
\tilde{\theta}^{i}=d X^{i}+X^{j} \tilde{\omega}_{j}^{i}
$$

As with the connection in the principal bundle $P\left(J^{r} M, G\right)$, the connection in the associated bundle $\mathcal{F}\left(P\left(J^{r} M, G\right)\right)$ is a connection which defines a representation of zero curvature for (1) as well. Therefore, the system of equations $\tilde{\theta}_{\Sigma}^{i}=0$, such that $\tilde{\theta}_{\Sigma}^{i}$ is the restriction of the form $\tilde{\theta}^{i}$ to the corresponding pull-back of the section $\Sigma \subset M$ defined by $u=u(x, y, \cdots)$, is completely integrable if and only if the section $\Sigma \subset M$ is a solution of (1).

This can be put in another way by using contact forms as principal forms. Then the $\omega_{j}^{i}$ will be equal to zero, and the forms $\tilde{\theta}^{i}$ assume the following structure,

$$
\tilde{\theta}^{i}=d X^{i}+X^{j} \Gamma_{j \lambda}^{i} \omega^{\lambda}
$$

where the $\omega^{\lambda}$ are one-forms on the base $M$. On a section, using (3) the equations $\tilde{\theta}_{\Sigma}^{i}=0$ will have the structure,

$$
d X^{i}+X^{j} \Gamma_{j 1}^{i}\left(x, y, u, u_{k}, \cdots\right) d x+X^{j} \Gamma_{j 2}^{i}\left(x, y, u, u_{k}, \cdots\right) d y=0 .
$$

Equation (7) has been written in terms of coordinates $(x, y)$ on the base manifold and when appropriate, $y$ can be identified as an evolution parameter $y=t$ in the examples.

\section{Bäcklund Transformations}

A Bäcklund transformation provides a type of mapping between different solutions to a pair of partial differential equations. The base space will be the same here for both equations. However, it will be required to consider jet spaces for each equation, which will be referred to as $J^{r} E_{1}$ and $J^{r} E_{2}$, respectively. Hence, $\left\{u, \lambda_{j_{1}, \cdots, j_{k}}\right\}$ constitute coordinates for the first space, and $\left\{v, \mu_{j_{1}, \cdots, j_{r}}\right\}$ constitute coordinates for the second space. It is said that a Bäcklund transformation is established between a pair of equations

$$
F_{1}\left(x, y, u, \lambda_{i}, \lambda_{j k}, \ldots\right)=0, \quad F_{2}\left(x, y, v, \mu_{i}, \mu_{j k}, \ldots\right)=0,
$$

provided that connections are defined in the principal bundles $P\left(J^{r} E_{1}, G\right)$ and $P\left(J^{r} E_{2}, G\right)$ which define representations of zero curvature for these equations. 
Moreover, in each of the manifolds $E_{1}$ and $E_{2}$, a structure on the bundle is defined with a one-dimensional associated fiber. For the case of $E_{2}$ it is with the principal bundle $P\left(J^{r} E_{1}, G\right)$. In the case of $E_{1}$, it is with the principal bundle $P\left(J^{r} E_{2}, G\right)$. The zero curvature connections which specify representations of zero curvature for the equations generate corresponding connections in the associated bundles $\mathcal{F}\left(P\left(J^{r} E_{1}, G_{1}\right)\right)$ and $\mathcal{F}\left(P\left(J^{r} E_{2}, G_{2}\right)\right)$ which specify representations of zero curvature with the connection forms $\theta$ and $\vartheta$, respectively. Then integral manifolds of the equation $\theta=0$ considered on pull-backs of solutions of $F_{1}=0$ are the pull backs of solutions of $F_{2}=0$. Similarly, the integral manifolds of $\vartheta=0$ considered on the pull backs of solutions to $F_{2}=0$ are the pull backs of solutions of $F_{1}=0$. Therefore, a correspondence between solutions of the two equations is achieved.

With respect to the group $G l(2)$, the forms $\theta$ and $\vartheta$ are taken to be

$$
\theta=d v-\xi_{j}^{i}(v) \tilde{\omega}_{i}^{j}=d v-a_{j}(v) \theta^{j},
$$

and,

$$
\vartheta=d u-\eta_{j}^{i}(u) \tilde{\pi}_{j}^{i}=d u-b_{j}(u) \vartheta^{j} .
$$

The structure forms on the right side of (9) and (10) are given as

$$
\tilde{\omega}_{j}^{i}=\omega_{j}^{i}+\Gamma_{j k}^{i} \omega^{k}, \quad \tilde{\pi}_{j}^{i}=\pi_{j}^{i}+\Phi_{j k}^{i} \omega^{k}, \quad i, j=1,2 .
$$

The forms $\theta^{i}$ and $\vartheta^{i}$ are then linear combinations of the structure forms $\tilde{\omega}_{i}^{j}$ and $\tilde{\pi}_{i}^{j}$, respectively. The coefficients $\Gamma_{j k}^{i}$ and $\Phi_{j k}^{i}$ in (11) are function-valued and depend on the coordinates of the base manifold and jet space. Thus (11) will constitute connection forms in the principal bundles, respectively.

If contact forms are selected as principal forms in the bundle of jets, then we can put $\omega_{j}^{i}=0$ and $\pi_{j}^{i}=0$. The set of forms (11) simplify to read,

$$
\tilde{\omega}_{j}^{i}=\Gamma_{j k}^{i}(x, y, u, \ldots) \omega^{k}, \quad \tilde{\pi}_{j}^{i}=\Phi_{j k}^{i}(x, y, v, \ldots) \omega^{k} .
$$

In this case, the two equations $\theta=0$ and $\vartheta=0$ considered on pull-backs of solutions of the equations $F_{1}=0$ and $F_{2}=0$ then take the form,

$$
d v-\xi_{j}^{i}(v) \Gamma_{i 1}^{j} d x-\xi_{j}^{i}(v) \Gamma_{i 2}^{j} d y=0, \quad d u-\eta_{j}^{i}(u) \Phi_{i 1}^{j} d x-\eta_{j}^{i}(u) \Phi_{i 2}^{j} d y=0 .
$$

This differential system is equivalent to the following systems of partial differential equations,

$$
v_{x}=\xi_{j}^{i}(v) \Gamma_{i 1}^{j}(x, y, u, \cdots), \quad v_{y}=\xi_{j}^{i}(v) \Gamma_{i 2}^{j}(x, y, u, \cdots),
$$


and

$$
u_{x}=\eta_{j}^{i}(u) \Phi_{i 1}^{j}(x, y, v, \cdots), \quad u_{y}=\eta_{j}^{i}(u) \Phi_{i 2}^{j}(x, y, v, \cdots) .
$$

The part of the formalism which determines whether this procedure will be productive is whether a system of differential forms $\theta^{i}$ and $\vartheta^{i}$, or equivalently $\tilde{\omega}_{j}^{i}$ and $\tilde{\pi}_{j}^{i}$ exist and can be found in closed form for the relevant equations. This implies that the forms $\theta^{i}$ or $\vartheta^{i}$ are defined so that the corresponding structure equations in terms of them produce at most nontrivial curvature terms which are related to the particular equation which has to vanish on a section. To keep the presentation straightforward, the simpler $G l(2)$ equations will be used. In terms of the forms $\theta^{i}$, they are written,

$$
\begin{gathered}
d \theta^{1}+\theta^{2} \wedge \theta^{3} \equiv R_{12} \omega^{1} \wedge \omega^{2}, \quad d \theta^{2}-\frac{1}{2} \theta^{1} \wedge \theta^{2} \equiv R_{112}^{2} \omega^{1} \wedge \omega^{2} \\
d \theta^{3}+\frac{1}{2} \theta^{1} \wedge \theta^{3} \equiv R_{212}^{1} \omega^{1} \wedge \omega^{2}
\end{gathered}
$$

The terms on the right hand side denote curvature terms, which may be present or not depending on how the forms $\theta^{i}$ are defined. When they appear in a nontrivial way, they will be related to the equation. A system analogous to (16) with the forms $\theta^{i}$ replaced by forms $\vartheta^{i}$ relevant to the second equation will hold as well. If such sets of forms can be found and written down, the procedure discussed will produce the required transformations by means of (14) and (15).

If the three one-forms $\theta^{i}$ and $\vartheta^{i}$ which appear in (9) and (10) are nontrivial and satisfy structure equations (16), then the following result holds.

Theorem 3.1. Suppose the nontrivial one-forms $\alpha^{i}$ satisfy structure equations (16). Then the one-form defined in terms of the $\alpha^{i}$ by

$$
\tau=d v-a(v) \alpha^{1}-b(v) \alpha^{2}-c(v) \alpha^{3}
$$

is closed provided the coefficients $a, b$ and $c$ satisfy the following system of first order equations

$$
a^{\prime} b-a b^{\prime}-\frac{1}{2} b=0, \quad c b^{\prime}-b c^{\prime}+a=0, \quad c a^{\prime}-a c^{\prime}+\frac{1}{2} c=0 .
$$

Proof. Differentiating (17) exteriorly, it is found that $d \tau=-a^{\prime}(v) d v \wedge \alpha^{1}-a(v) d \alpha^{1}-b^{\prime}(v) d v \wedge \alpha^{3}-b(v) d \alpha^{2}-c^{\prime}(v) d v \wedge \alpha^{3}-c(v) d \alpha^{3}$. 
Now $d v$ can be obtained from $\tau \equiv 0$ and structure equations (16) in terms of the $\alpha^{i}$ can be substituted into $d \tau$ assuming curvature terms are absent to obtain

$$
\begin{gathered}
d \tau=-b a^{\prime} \alpha^{2} \wedge \alpha^{1}-c a^{\prime} \alpha^{3} \wedge \alpha^{1}+a \alpha^{2} \wedge \alpha^{3}-a b^{\prime} \alpha^{1} \wedge \alpha^{2}-c b^{\prime} \alpha^{3} \wedge \alpha^{2}-\frac{b}{2} \alpha^{1} \wedge \alpha^{2} \\
-a c^{\prime} \alpha^{1} \wedge \alpha^{3}-b c^{\prime} \alpha^{2} \wedge \alpha^{3}+\frac{1}{2} c \alpha^{1} \wedge \alpha^{3} \\
=\left(b a^{\prime}-a b^{\prime}-\frac{1}{2} b\right) \alpha^{1} \wedge \alpha^{2}+\left(c b^{\prime}-b c^{\prime}+a\right) \alpha^{2} \wedge \alpha^{3}+\left(c a^{\prime}-a c^{\prime}+\frac{1}{2} c\right) \alpha^{1} \wedge \alpha^{3} .
\end{gathered}
$$

Equating the coefficients of the two-forms $\alpha^{i} \wedge \alpha^{j}$ to zero, the system of differential equations (18) follows and consequently $d \tau$ vanishes, and so $\tau$ is closed.

Corollary 3.1. There exists a solution to system (18) of the form

$$
a(v)=1, \quad b(v)=e^{-v / 2}, \quad c(v)=e^{v / 2} .
$$

Proof. To obtain solution (19), set $a=1$ in the system. The three equations then take the form $b^{\prime}+\frac{1}{2} b=0, c b^{\prime}-b c^{\prime}+1=0, c^{\prime}-\frac{1}{2} c=0$. The first and last of these equations are integrated and the integration constant is taken to be one yielding

$$
b(v)=e^{-v / 2}, \quad c(v)=e^{v / 2} .
$$

All three equations are satisfied, and so $\tau$ then has the specific form,

$$
\tau=d v-\alpha^{1}-e^{-v / 2} \alpha^{2}-e^{v / 2} \alpha^{3} .
$$

Replacing the $\alpha^{i}$ by $\theta^{i}$, the form $\theta$ is given as

$$
\theta=d v-\theta^{1}-e^{-v / 2} \theta^{2}-e^{v / 2} \theta^{3} .
$$

There is a similar form for the set of $\vartheta^{i}$. Of course, other solutions of (18) should exist and might be used to construct these objects to yield other different transformations as well.

\section{Applications of the Theory to Equations}

It has been one of the intentions at the outset to give a number of examples which illustrate several applications of the theory to obtain Bäcklund systems for some nonlinear equations. Of course, it is natural to group equations in 
pairs, but for conciseness, the equations will be presented consecutively in terms of just the $\left(u, \lambda_{l}, \tilde{\omega}_{j}^{i}\right)$ set of variables. To push on further, any other equation can be selected and the variables in the results written in terms of the $\left(v, \mu_{l}, \tilde{\pi}_{j}^{i}\right)$ system of variables and then combined with an equation in the other set of variables to constitute a pair which will be of further use. As the coordinates of the base manifold in each of the cases, we continue to refer to them as $(x, y)$ where $y=t$ can represent an evolution parameter. Let us then set $\omega^{1}=d x$ and $\omega^{2}=d y$ so $\tilde{\omega}_{j}^{i}$ takes the form (12). At the end, $y$ can be replaced by $t$ if it is intended to represent an evolution variable.

\subsection{The Korteweg de-Vries Equation}

The first equation that will be discussed is the Korteweg de-Vries (KdV) equation. Let $k$ be any real constant and define the set of structural forms

$$
\begin{gathered}
\tilde{\omega}_{1}^{1}=-\lambda_{1} \omega^{2}, \\
\tilde{\omega}_{2}^{1}=\omega^{1}+(4 k-2 u) \omega^{2}, \\
\tilde{\omega}_{1}^{2}=-(k+u) \omega^{1}+\left(\lambda_{11}-(k+u)(4 k-2 u)\right) \omega^{2}, \\
\tilde{\omega}_{2}^{2}=\lambda_{1} \omega^{2}
\end{gathered}
$$

The corresponding forms $\theta^{i}$ are defined in terms of the structural forms $\tilde{\omega}_{j}^{i}$ as

$$
\theta^{1}=2\left(\tilde{\omega}_{1}^{1}-\tilde{\omega}_{2}^{2}\right), \quad \theta^{2}=2 \tilde{\omega}_{1}^{2}, \quad \theta^{3}=-2 \tilde{\omega}_{2}^{1} .
$$

Substituting (21), these are

$$
\begin{gathered}
\theta^{1}=-4 \lambda_{1} \omega^{2}, \\
\theta^{2}=-(k+u) \omega^{1}+2\left(\lambda_{11}-(k+u)(4 k-2 u)\right) \omega^{2} \\
\theta^{3}=-2 \omega^{1}-2(4 k-2 u) \omega^{2} .
\end{gathered}
$$

Substituting (23) into structure equations (16), it is easy to obtain

$$
\begin{gathered}
d \theta^{1}+\theta^{2} \wedge \theta^{3}=-4 d \lambda_{1} \wedge \omega^{2}+4 \lambda_{1} \omega^{1} \wedge \omega^{2}, \\
d \theta^{2}-\frac{1}{2} \theta^{1} \wedge \theta^{2}=2\left(\lambda_{111}+6 u u_{1}+u_{2}\right) \omega^{1} \wedge \omega^{2}, \\
d \theta^{3}+\frac{1}{2} \theta^{1} \wedge \theta^{3}=4\left(u_{1}-\lambda_{1}\right) \omega^{1} \wedge \omega^{2} .
\end{gathered}
$$


The set of $\Gamma_{j k}^{i}$ can now be obtained from the set of $\tilde{\omega}_{j}^{i}$ as follows

$$
\begin{array}{cccc}
\Gamma_{11}^{1}=0, & \Gamma_{21}^{2}=0, & \Gamma_{11}^{2}=-(k+u), & \Gamma_{21}^{1}=1, \\
\Gamma_{12}^{1}=-\lambda_{1}, & \Gamma_{22}^{2}=\lambda_{1}, & \Gamma_{12}^{2}=\lambda_{11}-(k+u)(4 k-2 u), & \Gamma_{22}^{1}=4 k-2 u .
\end{array}
$$

Using (13) and (25), the form $\theta$ assumes the structure (20) where

$$
\xi_{1}^{1}=2, \quad \xi_{2}^{2}=-2, \quad \xi_{2}^{1}=2 e^{-v / 2}, \quad \xi_{1}^{2}=-2 e^{v / 2} .
$$

Upon restricting these quantities to a section the following theorem results

Theorem 4.1. A Bäcklund system for the $K d V$ equation is given by

$$
\begin{aligned}
& v_{x}=-2(k+u) e^{-v / 2}-2 e^{v / 2}, \\
& v_{y}=-4 u_{x}-4(2 k-u) e^{v / 2}+2\left(u_{x x}-(k+u)(4 k-2 u)\right) e^{-v / 2} .
\end{aligned}
$$

Proof. It is straightforward at this point to substitute the information generated thus far into system (14) to obtain (27). It remains to verify the compatibility condition. Upon differentiating $v_{x}$ and $v_{y}$ in (27), the following pair of derivatives is obtained

$$
\begin{gathered}
v_{x y}=-2 u_{y} e^{-v / 2}+(k+u) e^{-v / 2} v_{y}-e^{v / 2} v_{y} \\
v_{y x}=-4 u_{x x}+4 u_{x} e^{v / 2}-2(2 k-u) e^{v / 2} v_{x}+2\left(u_{x x x}-u_{x}(4 k-2 u)+2(k+u) u_{x}\right) e^{-v / 2} \\
\left.-v_{x}\left(u_{x x}-(k+u)(4 k-2 u)\right)\right) e^{-v / 2} .
\end{gathered}
$$

It is found that the constant $k$ disappears from the difference of these derivatives which is given by

$$
v_{x y}-v_{y x}=-2 e^{-v / 2}\left(u_{x x x}+6 u u_{x}+u_{y}\right) .
$$

This expression vanishes whenever $u$ satisfies the KdV equation

$$
u_{x x x}+6 u u_{x}+u_{y}=0 .
$$

\subsection{The Liouville Equation}

Let us apply this formalism to obtain system (14) for the Liouville equation $u_{x y}=e^{u}$. First the connection in the principal bundle must be defined which is to be of zero curvature. In this case, the connection forms in the principal 
bundle are defined as in (12). A system of forms which will work is given as follows

$$
\tilde{\omega}_{1}^{1}=-\frac{\lambda_{1}}{4} d x+\frac{\lambda_{2}}{4} d y, \tilde{\omega}_{2}^{2}=\frac{\lambda_{1}}{4} d x-\frac{\lambda_{2}}{4} d y, \tilde{\omega}_{1}^{2}=\frac{1}{\sqrt{2}} e^{u / 2} d x, \tilde{\omega}_{2}^{1}=\frac{1}{\sqrt{2}} e^{u / 2} d y .
$$

Based on these forms, the coefficients $\Gamma_{i k}^{j}$ are given as

$$
\begin{gathered}
\Gamma_{11}^{1}=-\frac{\lambda_{1}}{4}, \quad \Gamma_{21}^{2}=\frac{\lambda_{1}}{4}, \quad \Gamma_{11}^{2}=\frac{1}{\sqrt{2}} e^{u / 2}, \quad \Gamma_{21}^{1}=0, \\
\Gamma_{12}^{1}=\frac{\lambda_{2}}{4}, \quad \Gamma_{22}^{2}=-\frac{\lambda_{2}}{4}, \quad \Gamma_{12}^{2}=0, \quad \Gamma_{22}^{1}=\frac{1}{\sqrt{2}} e^{u / 2} .
\end{gathered}
$$

The corresponding forms $\theta^{i}$ are defined in terms of the structural forms $\tilde{\omega}_{j}^{i}$ by means of (22),

$$
\theta^{1}=-\lambda_{1} \omega^{1}+\lambda_{2} \omega^{2}, \quad \theta^{2}=\sqrt{2} e^{u / 2} \omega^{1}, \quad \theta^{3}=-\sqrt{2} e^{u / 2} \omega^{2} .
$$

It can be shown by explicit calculation that the forms (22) determine a representation of zero curvature for the Liouville equation. To do this, it is straightforward to work out structure equations (16) and they take the form

$$
\begin{gathered}
d \theta^{1}+\theta^{2} \wedge \theta^{3}=-d \lambda_{1} \wedge d \omega^{1}+d \lambda_{2} \wedge d \omega^{2}-2 e^{u} d \omega^{1} \wedge d \omega^{2}, \\
d \theta^{2}-\frac{1}{2} \theta^{1} \wedge \theta^{2}=\frac{1}{\sqrt{2}} e^{u / 2}\left(d u-\lambda_{2} \omega^{2}\right) \wedge \omega^{1} \\
d \theta^{3}+\frac{1}{2} \theta^{1} \wedge \theta^{3}=\frac{1}{\sqrt{2}} e^{u / 2}\left(-d u+\lambda_{1} \omega^{1}\right) \wedge \omega^{2} .
\end{gathered}
$$

Note that curvature terms appear on the right hand side. However, on a section $\Sigma_{1} \subset E_{1}$, by (3), it follows that all of these vanish and hence $\lambda_{1}=u_{x}, \lambda_{2}=u_{y}$. The first equation is satisfied provided that $u$ satisfies the equation $u_{x y}=e^{u}$. The $\xi_{j}^{i}$ can again be given as in (26) hence $d \theta$ takes the form (20). Combining these results we have the following theorem.

Theorem 4.2. Connection (29) with (26) and system (14) result in the following Bäcklund system

$$
u_{x}+v_{x}=\sqrt{2} e^{(u-v) / 2}, \quad u_{y}-v_{y}=\sqrt{2} e^{(u+v) / 2} .
$$

Proof. Substituting (26) and (30) into (14), the system summarized in (32) appears. It is again straightforward to verify the compatibility condition for system (32). 


\subsection{The Burgers Equation}

In order to apply the method effectively, a set of forms for the structure equations must be produced. As this may not always be easy, it is worth considering some examples where only two nontrivial forms appear. In order to do this, it may at times be necessary to formally integrate over one of the jet variables, in this case $u$. One way to obtain the Burgers equation is to begin with the set of structural forms given by

$$
\tilde{\omega}_{1}^{1}=\frac{1}{2} \omega^{2}, \tilde{\omega}_{1}^{2}=-\frac{1}{2} u \omega^{1}+\frac{1}{2}\left(\lambda_{1}+u^{2}+\int_{x_{0}}^{x} u d s\right) \omega^{2}, \tilde{\omega}_{2}^{1}=0, \tilde{\omega}_{2}^{2}=-\frac{1}{2} \omega^{2} .
$$

Based on the structural forms (33), the set of forms $\theta^{i}$ then follow

$$
\theta^{1}=2 \tilde{\omega}_{1}^{2}, \quad \theta^{2}=-u \omega^{1}+\left(\lambda_{1}+u^{2}+\int_{x_{0}}^{x} u d s\right) \omega^{2}, \quad \theta^{3}=0 .
$$

Now only two of the structure equations survive and on a section,

$$
d \theta^{1}+\theta^{2} \wedge \theta^{3}=0, \quad d \theta^{2}-\frac{1}{2} \theta^{1} \wedge \theta^{2}=\left(\left(\lambda_{1}+u^{2}\right)_{1}+u_{2}\right) \omega^{1} \wedge \omega^{2} .
$$

The $\Gamma_{j k}^{i}$ can be obtained from these forms, and with just two nontrivial $\theta^{i}$ the set of $\xi_{j}^{i}$ are taken to be $\xi_{1}^{1}=-2, \xi_{2}^{2}=2$ and $\xi_{2}^{1}=e^{v / 2}$. The compatibility will continue to hold and equations (14) yield the following theorem.

Theorem 4.3. System (14) corresponding to the forms (34) is given as

$$
v_{x}=-\frac{u}{2} e^{v / 2}, \quad v_{y}=-2+\frac{1}{2} e^{v / 2}\left(u_{x}+u^{2}+\int_{x_{0}}^{x} u d s\right) .
$$

Proof. It remains to study the compatibility relation. To do this the derivatives of $v_{x}$ and $v_{y}$ in (35) are required,

$$
\begin{aligned}
& v_{x y}=-\frac{1}{2} e^{v / 2}\left(u_{y}+\frac{u}{2}\left(-2+\frac{e^{v / 2}}{2}\left(u_{x}+u^{2}+\int_{x_{0}}^{x} u d s\right)\right)\right), \\
& v_{y x}=\frac{1}{2}\left(-\frac{u}{4} e^{v / 2}\left(u_{x}+u^{2}+\int_{x_{0}}^{x} u d s\right)+u_{x x}+2 u u_{x}+u\right) .
\end{aligned}
$$

Equating the derivatives and simplifying, the compatibility relation is found to hold provided that $u$ satisfies the equation

$$
u_{x x}+2 u u_{x}+u_{y}=0 .
$$




\subsection{A Generalized KdV Equation}

This equation is a generalization of the $\mathrm{KdV}$ equation which has been considered above. It has received some considerable attention recently [16]. Here, it will be shown that it can be handled with two structure equations. To begin this, define the structural forms as follows,

$\tilde{\omega}_{1}^{1}=\frac{1}{2} \omega^{2}, \tilde{\omega}_{1}^{2}=-\frac{1}{2} u \omega^{1}+\frac{1}{2}\left(\lambda_{11}+\frac{\beta}{n+1} u^{n+1}+\int_{x_{0}}^{x} u d s\right) \omega^{2}, \tilde{\omega}_{2}^{1}=0, \tilde{\omega}_{2}^{2}=-\frac{1}{2} \omega^{2}$.

In (36), $\beta$ is a real nonzero constant. It is of interest to compare forms (36) to the ones used to study the Burgers equation. The set of one-forms $\theta^{i}$ are then given as

$$
\theta^{1}=2 \omega^{2}, \quad \theta^{2}=-u \omega^{1}+\left(\lambda_{11}+\frac{\beta}{n+1} u^{n+1}+\int_{x_{0}}^{x} u d s\right) \omega^{2}, \quad \theta^{3}=0 .
$$

These forms are put into structure equations (16) to yield,

$$
d \theta^{1}+\theta^{2} \wedge \theta^{3}=0, \quad d \theta^{2}-\frac{1}{2} \theta^{1} \wedge \theta^{2}=\left(\left(\lambda_{11}+\frac{\beta}{n+1} u^{n+1}\right)_{1}+u_{2}\right) \omega^{1} \wedge \omega^{2} .
$$

The necessary information with regard to the required $\Gamma_{j k}^{i}$ can be taken from these forms and the $\xi_{j}^{i}$ used will be the same as in the previous example. Combining all of this information, system (14) yields the following theorem.

Theorem 4.4. System (14) corresponding to the system of forms (36) is given explicitly as

$$
v_{x}=-\frac{1}{2} u e^{v / 2}, \quad v_{y}=-2+\frac{1}{2} e^{v / 2}\left(u_{x x}+\frac{\beta}{n+1} u^{n+1}+\int_{x_{0}}^{x} u d s\right) .
$$

Proof. The compatibility condition relies on the following derivatives which follow from (39),

$$
\begin{gathered}
v_{x y}=-\frac{1}{2}\left(u_{y}+\frac{u}{2} v_{y}\right) e^{u / 2}, \\
v_{y x}=\frac{1}{4} v_{x} e^{v / 2}\left(u_{x x}+\frac{\beta}{n+1} u^{n+1}+\int_{x_{0}}^{x} u d s\right)+\frac{1}{2} e^{v / 2}\left(\left(u_{x x}+\frac{\beta}{n+1} u^{n+1}\right)_{x}+u\right) .
\end{gathered}
$$

Equating these derivatives, simplifying and collecting all terms on one side, the compatibility condition is found to hold provided that the following generalized $\mathrm{KdV}$ equation applies

$$
u_{x x x}+\beta u^{n} u_{x}+u_{y}=0 .
$$

This equation is exactly the one produced by the structure equations (38) using the forms (37). 


\section{References}

[1] H. D. Wahlquist and F. B. Estabrook, Prolongation structures of nonlinear evolution equations, J. Math. Phys. 16, (1975), 1-7.

[2] F. B. Estabrook and H. D. Wahlquist, Prolongation structures of nonlinear evolution equations II, J. Math. Phys. 17, (1976), 1293-1297.

[3] F. B. Estabrook, Moving frames and prolongation algebras, J. Math. Phys. 23, (1982), 2071-2076.

[4] F. B. Estabrook, Bäcklund Transformations the Inverse Scattering Method, Solitons and Their Applications, Lecture Notes in Mathematics, ed. R. Miura, vol. 515, Springer, Berlin, (1976).

[5] R. Hermann, Pseudodifferentials of Estabrook and Wahlquist, the geometry of solutions and the theory of connections, Phys. Rev. Letts. 36, (1976), 835-836.

[6] R. Hermann, The Geometry of NonLinear Differential Equations, Bäcklund Transformations and Solitons, vol. XII, A, Math. Sci. Press, Brookline, MA, (1976).

[7] M. J. Ablowitz, D. K. Kaup, A. C. Newell and H. Segur, Nonlinear evolution equations of physical significance, Phys. Rev. Letts. 31, (1973), 125127.

[8] M. J. Ablowitz and H. Segur, Solitons and the Inverse Scattering Transform, SIAM, Studies in Applied Mathematics, Philadelphia, PA, (1981).

[9] P. Bracken, Connections of Zero Curvature and Applications to Nonlinear Partial Differential Equations, Discrete and Continuous Dynamical Systems, Series S, 7, 6, (2014), 1165-1179.

[10] P. Bracken, A Geometric Interpretation of Prolongation by Means of Connections, J. Math. Phys. 51, (2010), 113502.

[11] P. Bracken, Exterior Differential Systems Prolongations and Application to a Study of Two Nonlinear Partial Differential Equations, Acta Appl. Math. 113, (2011), 247-263.

[12] E. van Groesen and E. M. Jager, Mathematical structures in continuous dynamical systems, Studies in Math. Physics, vol. 6, North Holland, Amsterdam, II, Ch. 6, (1994). 
[13] J. Krasilshchik and A. Verbovetsky, Geometry of Jet Spaces and Integrable Systems, J. Geom. and Physics, 61, (2011), 1633-1674.

[14] A. K. Rybnikov, Connections Defining Representations of Zero Curvature and the Solitons of sine-Gordon and Korteweg-de Vries Equations, Russian J. of Math. Phys. 18, (2011), 195-210.

[15] A. K. Rybnikov, Equations of the Inverse Problem, Bäcklund Transformations and the Theory of Connections, J. of Math. Sciences, 94, (2009), 1685-1699.

[16] P. Bracken, Connections Defining Representations of Zero Curvature and their Lax and Bäcklund Mappings, Journal of Geometry and Physics, 70, (2013), 157-163. 